\title{
Chronic Disease Stigma, Skepticism of the Health System, and Socio-economic Fragility: Qualitative Assessment of Factors Impacting Receptiveness to Group Medical Visits and Microfinance for Non- Communicable Disease Care in Rural Kenya
}

Rae Dong

Icahn School of Medicine at Mount Sinai

Claudia Leung

Duke University Hospital

Mackenzie N. Naert

Icahn School of Medicine at Mount Sinai

Violet Naanyu

Moi University

Peninah Kiptoo

AMPATH Kenya

Winnie Matelong

AMPATH Kenya

Esther Matini

AMPATH Kenya

Vitalis Orango

AMPATH Kenya

Gerald S. Bloomfield

Duke University School of Medicine

David Edelman

Duke University School of Medicine

Valentin Fuster

Icahn School of Medicine at Mount Sinai

Simon Manyara

AMPATH Kenya

Diana Menya

Moi University

Sonak D. Pastakia

Purdue University 


\section{Tom Valente}

Keck Hospital of USC

Jemima Kamano

Moi University

Carol R. Horowitz

Icahn School of Medicine at Mount Sinai

Rajesh Vedanthan ( $\nabla$ rajesh.vedanthan@nyulangone.org )

NYU Langone Health https://orcid.org/0000-0001-7138-2382

\section{Research}

Keywords: group medical visits, microfinance, hypertension, diabetes, qualitative inquiry

Posted Date: July 24th, 2020

DOI: https://doi.org/10.21203/rs.3.rs-47902/v1

License: (c) (i) This work is licensed under a Creative Commons Attribution 4.0 International License.

Read Full License 


\section{Abstract}

Background: Non-communicable diseases (NCDs) are the leading cause of mortality in the world, and innovative approaches to NCD care delivery are being actively developed and evaluated. Combining the group-based experience of microfinance and group medical visits is a novel approach to NCD care delivery that has demonstrated beneficial impact in previous pilot studies. However, the contextual factors, facilitators, and barriers impacting wide-scale implementation of these approaches within a lowand middle-income country setting are not well known.

Methods: Two types of qualitative group discussion were conducted: 1) mabaraza (singular, baraza), a traditional East African community gathering used to discuss and exchange information in large group settings; and 2) focus group discussions (FGDs) among rural clinicians, community health workers, microfinance group members, and patients with NCDs. Trained research staff members led the discussions using structured question guides. Content analysis was performed with NVivo using deductive and inductive codes that were then grouped into themes.

Results: We conducted 5 mabaraza and 16 FGDs. A total of 205 individuals (113 men and 92 women) participated in the mabaraza, while 162 individuals (57 men and 105 women) participated in the FGDs. Participants expressed interest in participating in microfinance and group medical visits, but cited several key challenges: 1) stigma of chronic disease, 2) earned skepticism of the health system, and 3) socioeconomic fragility.

Conclusions: Our qualitative study revealed and illuminated actionable factors that could impact the success of implementation of group medical visits and microfinance initiatives for NCD care. While several challenges were highlighted, participants also felt that planned interventions could address and mitigate the impact of these dynamic factors. We anticipate that our approach and analysis provides new insights and methodological techniques that will be relevant to other low-resource settings worldwide.

Trial Registration: Clinicaltrials.gov, NCT02501746, registration date: July 17, 2015

\section{Contributions To The Literature}

1. Combining the group-based experience of microfinance and group medical visits is a novel approach to NCD care delivery.

2. Our qualitative study revealed that stakeholders in western Kenya were interested in microfinance and group medical visits, but cited several key challenges: 1) stigma of chronic disease, 2) skepticism of the health system, and 3) socio-economic fragility.

3. This type of formative work helps to illuminate factors that can positively and negatively impact the remainder of the implementation science study.

4. Our methods included novel qualitative approaches (e.g. mabaraza, traditional community gatherings) and involved multiple stakeholder groups, allowing us to triangulate multiple 
perspectives.

\section{Background}

Non-communicable diseases (NCDs) are the leading cause of mortality in the world, with $80 \%$ of this burden occurring in low- and middle-income countries (LMICs) (1). Innovative approaches to NCD care delivery are being actively developed and evaluated. In particular, there is increasing recognition that social determinants of health need to be incorporated into care delivery, in order to simultaneously address socio-economic as well as health issues $(2,3)$.

One potentially promising approach includes microfinance (MF) initiatives, which are financial services targeted at individuals, groups of individuals, or small businesses, to provide individuals with access to saving mechanisms and loan opportunities (4-7). MF activities have been shown to reduce poverty and improve health outcomes (8). Another innovative care delivery approach is the group medical visit (GMV), which is a clinical encounter involving a group of patients, and has been shown to increase the efficiency of care delivery, quality of care, enhance social support, and encourage self-efficacy $(9,10)$. Combining the group-based experience of MF with a GMV is a novel approach to NCD care delivery that has demonstrated beneficial impact in a small pilot study in western Kenya (11). However, the contextual factors, facilitators, and barriers impacting wide-scale implementation of these approaches within an LMIC setting are not well studied.

The Bridging Income Generation with GrouP Integrated Care (BIGPIC) study in western Kenya is evaluating the impact of MF and GMVs on cardiovascular risk reduction among individuals with and at increased risk of diabetes.(12) The formative phase of this study aimed to identify the contextual factors, facilitators, and barriers that may impact the success this approach. In this paper, we report the results of that pre-implementation formative inquiry.

\section{Methods}

\section{Setting}

The Academic Model Providing Access to Healthcare (AMPATH) is a partnership between Moi University College of Health Sciences, Moi Teaching and Referral Hospital (both in western Kenya), and a consortium of North American academic medical centers (13). AMPATH established a system of care delivery for HIV patients in 2001. Subsequently, in response to the growing burden of chronic disease (particularly diabetes and hypertension) within the population (14), expanded its clinical scope to include primary health care and chronic disease management serving a catchment area of over 4 million people (15). The chronic disease management program primarily provides health facility-based care for patients with diabetes and hypertension.

\section{Participants and procedures}


For this qualitative study, community members were invited to join mabaraza (singular, baraza), a traditional East African community gathering used to discuss and exchange information regarding a variety of topics and issues in large group settings (16). In addition, we conducted focus group discussions (FGDs) of 10-15 participants each, among people with shared characteristics (e.g. rural clinicians, community health workers, microfinance group members, and patients with chronic diseases). Structured question guides were developed to include content related to experience of chronic disease care, perceptions of MF and GMVs, and factors that might impact joining and remaining in groups. These question guides were pilot-tested on community members, patients, and clinicians prior to being used in the qualitative sessions. Trained research staff members led the discussions in English and Kiswahili, as was appropriate for the participants.

Sessions were audio-recorded, transcribed, and translated to English. Content analysis was performed with NVivo using deductive and inductive codes that were then grouped into themes. A kappa score of $>$ 0.90 was established as the threshold to ensure inter-rater reliability among three independent coders (RD, $\mathrm{CL}, \mathrm{MN})$. The Standards for Reporting Qualitative Research checklist was completed (Additional File) (17).

\section{Results}

In total, 21 qualitative sessions ( 5 mabaraza and 16 FGDs) were conducted in 11 distinct geographic regions in western Kenya. A total of 205 individuals (113 men and 92 women) participated in the mabaraza, while 162 individuals (57 men and 105 women) participated in the FGDs. Participants expressed interest in participating in MF/GMV programs, but cited several key challenges. Specifically, participants described stigma of chronic disease, earned skepticism of the health system, and socioeconomic fragility as major barriers to MF and GMVs for NCDs.

Stigma of Chronic Disease

Participants noted that, specific to NCDs, there was the potential for stigma and being considered a distinct 'other,' characterized by undesirable status or negative stereotypes. For example, some participants described patients with hypertension as lacking motivation to improve or get better. With respect to group-based MF or GMV, there was concern that membership in the group would lead to being labeled as "sick" and potentially "inferior."

Maybe the disadvantage can come in the form of stigma where outsiders can christen the group the title 'people with pressure,' the group would be known by such a title.

Conversely, some participants expressed optimism that participation in MF or GMV could increase a sense of "belonging," acceptance, and social cohesion, which could counter the potential for negative stigma. 
There is also stigma reduction when they are in groups, someone feels that they are not alone with this condition.

Skepticism of the Health System

Skepticism of the health system was described regarding both the overall quality of care provided, as well as trust in clinical providers. Much of this skepticism was grounded in participants having had previous negative experiences with the health system and clinicians. Participants reported experiencing a lack of respect, verbal abuse, and not getting adequate or comprehensive services.

There was a time I was taken there it's like I saw the devil with my naked eyes! The kind of verbal abuse you get there! And also beating! You will be very surprised ... until I wondered and decided if this was a hospital facility really.

The problem affecting the community, most people are afraid of going to hospital, the way of approach, the way the doctor communicates, the way he starts, let alone serving you, the way he enters and welcomes you contributes for a person to fear the doctor.

There were some notable differences in previous experiences in the public vs. private sector, but neither sector was free from criticism or concern. For instance, participants reported that in the private sector, doctors' actions are felt to be driven by money and commercial interests, and they might not have patients' best interests at heart. In contrast, public-sector health providers who are paid a salary are not incentivized to provide services for the purposes of making more money. These providers were described as being "serious" and "more professional." However, participants also reported the opposite experience, where private-sector providers were seen as providing higher quality care because they are incentivized to treat patients better in order to increase their income, in contrast to public doctors who are not necessarily incentivized to provide quality care in this way. Private sector health facilities were also viewed as being more efficient and clean, but more expensive than the public sector, which was described as being less expensive but of poor quality.

I am not paid according to the patients I serve, if I treat just one or two I will still get my salary.

Some like private [facilities] because of the fast services. When you reach there it does not take time even though it is a bit expensive but your time will be shorter.

GMV, in particular, and MF were felt to have the potential to lead to increased clinician engagement and accountability. Given that a group of patients would be together for a GMV, participants felt that the clinician would be more responsive, more respectful, and more accountable.

I also support a group, is very important because it will make the doctor to work harder, unlike one by one.

In addition, it was felt that MF and GMV would increase both social and instrumental support with respect to access to care. Specifically, the group-based format could serve as an avenue for advocacy 
and for increasing the confidence to advocate on behalf of oneself and other group members.

When they are together and they teach each other they also motivate themselves, and the groups will help them if there are other needs. They can get money in the groups [...] or maybe there is a certain drug missing and they can get to work with the doctor and tells them it is this amount so they can go as a group and bargain for the cost to go down.

Socio-Economic Fragility

Study participants described a nearly all-encompassing sense of socioeconomic fragility that adversely impacted the entire care cascade, from being screened to seeking care to affording medications to completing follow-up visits. For example, lack of access to medicines due to cost was considered a major barrier to experiencing positive health outcomes.

When asked, 'Why didn't you come early?' They say, 'I was trying to look for money.'

In addition, poor health and unplanned illness were felt to further exacerbate an individual's and family's economic strain due to the cost of medical care, as well as lost wages.

Diseases don't tell when they come. It can be even at night. Now at night, where will you go to look for money-nowhere. Your work is to wait till morning for you to go and borrow.

That time when you are sick, the time you go to the hospital it means, like if you have the jobs that you do, you will not progress, so when you see you are just at the hospital, your income has stopped because you will not be working.

Socio-economic fragility was felt to worsen the impact of previously described stigma and health system skepticism. Participants reported that challenges with health care access due to affordability would adversely affect both real and perceived quality of care received by patients. In a negatively reinforcing cycle, the poorer quality of care would exacerbate health system skepticism, leading to lower healthcare utilization, delayed care-seeking, and lower adherence to medical advice, resulting in even worse health outcomes.

The combination of GMV and MF were felt to hold promise for addressing this socio-economic fragility. MF was felt to directly increase liquidity and purchasing capacity, and indirectly to improve overall income-earning potential. In addition, GMV was felt to potentially increase social support and thereby increase motivation and capacity for economic improvement.

Group is good because when the money gets to the table we are happy even though you don't own [it] all but we can divide equally and use them for hospital expenses.

When you are together, you will find that your colleague is better off and you give yourself hope. You now get energized. Now, another time you come together, you may find that another colleague has changed a bit and improved. As time goes by, you will find that every member in the group becomes strong. 


\section{Discussion}

In this qualitative study from western Kenya, we found that chronic disease stigma, skepticism of the health system, and socio-economic fragility were all factors that could impact the potential implementation success of GMV and MF for patients with diabetes and hypertension (Figure). Importantly, all three factors were reported as potential barriers for any NCD program and were based on historical experiences that did not necessarily include previous exposure to GMV or MF. Conversely, participants also felt that GMV and MF could potentially address and mitigate the impact of these dynamic factors.

Stigma has commonly been associated with infectious diseases such as HIV, and HIV-related stigma and discrimination have been well established as barriers to accessing HIV prevention, treatment, and support services (18). Our group has previously reported that co-locating hypertension management in the same facility as HIV care can present challenges due to HIV-related stigma (19). However, in the current study, participants described NCD-specific stigma that could act as a barrier to care. Others have reported that individuals with NCDs feel like they are blamed for their own illness by community members and health care workers (20). In particular, individuals who anticipated greater stigma from health care workers have been found to be less likely to access health care due to the prior negative experiences (21). Specific to the group-based GMV and MF activities proposed in this study, stigma may lead to fear of joining a patient group because being linked to the group may be associated with negative stereotyping, lower social status, and discrimination.

Stigma related to health care workers' attitudes towards patients with NCDs may contribute to the health system skepticism described by participants, as described above. In addition, participants reported instances of verbal abuse and lack of being respectfully treated by health care staff. Perceived low quality of care has been corroborated by empirical data indicating poor quality of care in LMICs (22). The adverse experiences described by our participants led to skepticism, lack of confidence, and lack of trust in the health system, which again has been widely reported in other parts of the world (23). Unfortunately, mistrust in clinical providers can lead to lower adherence to medical advice and subsequent poor health outcomes $(24,25)$. Skepticism of the health system has also been associated with lower health care utilization, lower rates of adoption of prevention interventions, and higher rates of unhealthy behaviors (26). This self-perpetuating, negatively reinforcing cycle yields adverse outcomes for individuals, populations, and health systems (27). Thus, it is imperative to break this cycle by improving quality of care, re-gaining trust of patients and community members, and disseminating these successes to the broader population.

Socio-economic fragility, in our population, appeared to exacerbate the potential negative sequelae of stigma and health care skepticism. Low socio-economic status is known to be associated with increased morbidity and mortality, although the mechanisms responsible for this are not fully established (28). In Kenya specifically, it has been demonstrated that poorer households in rural areas are more likely to experience catastrophic out-of-pocket expenses, primarily related to payments for outpatient services 
(29). At the societal level, socio-economic inequality is associated with disparities in NCD burden (30). In our setting, all of the above dynamics appeared to be relevant. We have previously described substantial levels of material deprivation and lack of health insurance in western Kenya (31), thus lending support to care delivery models, such as BIGPIC, that incorporate social determinants of health into clinical care (11).

Participants in general felt that, despite the barriers presented by stigma, skepticism, and socio-economic fragility, the combination of GMV and MF could potentially address those barriers and be successful despite those factors. Given that this qualitative inquiry was the formative component of a larger implementation research trial (12), we have been vigilant to incorporate the findings from this inquiry into the design of the BIGPIC intervention using a stakeholder-based, human-centered design process (32). At the same time, we recognize that our planned intervention will not be able to fully solve all of the potential issues, such as poverty and lack of health insurance. We are therefore heartened by the rollout and scaleup of universal health coverage programs in Kenya and other LMICs, which will provide much-needed financial risk protection for these populations (33).

We acknowledge the following limitations of our study. First, while we attempted to involve multiple stakeholder groups, it is likely that not all stakeholder perspectives were fully represented in this qualitative study. The overall BIGPIC project has other components that involve stakeholder engagement, such as the human-centered design process, in order to secure broader and deeper stakeholder participation throughout the implementation research project. Second, we recognize the potential for limited generalizability, since we recruited participants from specific geographic areas in western Kenya. Several of the salient themes, however, are consistent with findings from literature arising from other geographies, as discussed above, thus indicating that elements are indeed relevant for similar settings worldwide. Third, we did not record individual-level demographic information for the quotations and transcript. However, we view the themes as arising from a collective discussion, not necessarily from any one specific individual.

\section{Conclusions}

NCDs are the leading cause of mortality in the world, and there is increasing recognition of the need to simultaneously address socio-economic as well as health issues in NCD management. Qualitative inquiry, as we have conducted in this study, is helpful to reveal and illuminate factors that may positively and negatively impact implementation success. The factors highlighted in our analysis-chronic disease stigma, skepticism of the health system, and socio-economic fragility-have clearly informed the design, development, and implementation of our group-based GMV and MF strategies for optimizing NCD management in western Kenya. We anticipate that our approach and analysis provides new insights and methodological techniques that may be relevant to other low-resource settings worldwide.

\section{Abbreviations}


AMPATH

Academic Model Providing Access to Healthcare

BIGPIC

Bridging Income Generation with GrouP Integrated Care

FGD

Focus group discussion

GMV

Group medical visit

LMICs

Low- and middle-income countries

MF

Microfinance

NCDs

Non-communicable diseases

\section{Declarations}

\section{Ethics approval and consent to participate:}

Ethics approval was obtained from NYU Grossman School of Medicine Institutional Review Board, Icahn School of Medicine Institutional Review Board, and Moi University Institutional Research and Ethics Committee. All participants provided verbal informed consent prior to participating in the study.

\section{Consent for publication:}

Not applicable

\section{Availability of data and materials:}

The datasets used and/or analysed during the current study are available from the corresponding author on reasonable request. This study complies with the NIH Public Access Policy, which ensures that the public has access to the published results of $\mathrm{NIH}$ funded research, and therefore, all results have been (and will be made) available from final peer-reviewed journal manuscripts (including this one) via the digital archive PubMed Central upon acceptance for publication.

\section{Competing interests:}

The authors declare that they have no competing interests

\section{Funding:}


Research reported in this publication was supported by the National Heart, Lung, and Blood Institute of the National Institutes of Health under award number R01HL125487. The content is solely the responsibility of the authors and does not necessarily represent the official views of the National Institutes of Health.

\section{Contributions}

to the literature

\section{Acknowledgements:}

The authors wish to thank the BIGPIC research team for incredible work and passion, and for all study participants for devoting their time and energy that led to this manuscript.

\section{References}

1. Roth GA, Abate D, Abate KH, Abay SM, Abbafati C, Abbasi N, et al. Global, regional, and national agesex-specific mortality for 282 causes of death in 195 countries and territories, 1980\&\#x2013;2017: a systematic analysis for the Global Burden of Disease Study 2017. The Lancet. 2018;392(10159):1736-88.

2. Gurewich D, Garg A, Kressin NR. Addressing Social Determinants of Health Within Healthcare Delivery Systems: a Framework to Ground and Inform Health Outcomes. Journal of general internal medicine. 2020;35(5):1571-5.

3. National Academies of Sciences E, Medicine. Integrating Social Care into the Delivery of Health Care: Moving Upstream to Improve the Nation\&\#039;s Health. Washington, DC: The National Academies Press; 2019. 194 p.

4. Lorenzetti LMJ, Leatherman S, Flax VL. Evaluating the effect of integrated microfinance and health interventions: an updated review of the evidence. Health policy and planning. 2017;32(5):732-56.

5. Gertler P, Levine DI, Moretti E. Do microfinance programs help families insure consumption against illness? Health Econ. 2009;18(3):257-73.

6. Association SEWs. Sewa Services 2009 [Available from: http://www.sewa.org/Sewa_Services.asp.

7. Gutiérrez M. Las microfinanzas. El sistema financiero en Guatemala.; 2009 July 2009.

8. Kim J, Ferrari G, Abramsky T, Watts C, Hargreaves J, Morison L, et al. Assessing the incremental effects of combining economic and health interventions: The IMAGE study in South Africa2009. 824$32 \mathrm{p}$.

9. Edelman D, McDuffie JR, Oddone E, Gierisch JM, Nagi A, Williams JW, Jr. VA Evidence-based Synthesis Program Reports. Shared Medical Appointments for Chronic Medical Conditions: A Systematic Review. Washington (DC): Department of Veterans Affairs (US); 2012. 
10. Jaber R, Braksmajer A, Trilling JS. Group visits: a qualitative review of current research. Journal of the American Board of Family Medicine : JABFM. 2006;19(3):276-90.

11. Pastakia SD, Manyara SM, Vedanthan R, Kamano JH, Menya D, Andama B, et al. Impact of Bridging Income Generation with Group Integrated Care (BIGPIC) on Hypertension and Diabetes in Rural Western Kenya. Journal of general internal medicine. 2017;32(5):540-8.

12. Vedanthan R, Kamano JH, Lee H, Andama B, Bloomfield GS, DeLong AK, et al. Bridging income generation with group integrated care for cardiovascular risk reduction: Rationale and design of the BIGPIC study. American heart journal. 2017;188:175-85.

13. Einterz RM, Kimaiyo S, Mengech HNK, Khwa-Otsyula BO, Esamai F, Quigley F, et al. Responding to the HIV Pandemic: The Power of an Academic Medical Partnership. 2007;82(8):812-8.

14. Pastakia SD, Cheng SY, Kirui NK, Kamano JH. Dynamics, Impact, and Feasibility of Self-Monitoring of Blood Glucose in the Rural, Resource-Constrained Setting of Western Kenya. Clinical diabetes : a publication of the American Diabetes Association. 2015;33(3):136-43.

15. Genberg B, Wachira J, Kafu C, Wilson I, Koech B, Kamene R, et al. Health System Factors Constrain HIV Care Providers in Delivering High-Quality Care: Perceptions from a Qualitative Study of Providers in Western Kenya. 2019;18:2325958218823285.

16. Naanyu V, Sidle JE, Frankel RM, Ayuku D, Nyandiko WM, Inui TS. Rooting inquiry in tradition: the health baraza as a tool for social research in Kenya. Qual Health Res. 2011;21(1):14-26.

17. O'Brien BC, Harris IB, Beckman TJ, Reed DA, Cook DA. Standards for reporting qualitative research: a synthesis of recommendations. Acad Med. 2014;89(9):1245-51.

18. Mahajan AP, Sayles JN, Patel VA, Remien RH, Sawires SR, Ortiz DJ, et al. Stigma in the HIV/AIDS epidemic: a review of the literature and recommendations for the way forward. AIDS (London, England). 2008;22 Suppl 2(Suppl 2):S67-S79.

19. Vedanthan R, Tuikong N, Kofler C, Blank E, Kamano JH, Naanyu V, et al. Barriers and Facilitators to Nurse Management of Hypertension in Rural Western Kenya: A Qualitative Analysis. Ethnicity and Disease. 2016;26:315-22.

20. Sayles JN, Wong MD, Kinsler JJ, Martins D, Cunningham WE. The association of stigma with selfreported access to medical care and antiretroviral therapy adherence in persons living with HIV/AIDS. Journal of general internal medicine. 2009;24(10):1101-8.

21. Earnshaw VA, Quinn DM. The Impact of Stigma in Healthcare on People Living with Chronic Illnesses. Journal of health psychology. 2011;17(2):157-68.

22. Berendes S, Heywood P, Oliver S, Garner P. Quality of Private and Public Ambulatory Health Care in Low and Middle Income Countries: Systematic Review of Comparative Studies. PLOS Medicine. 2011;8(4):e1000433.

23. Kruk ME, Gage AD, Arsenault C, Jordan K, Leslie HH, Roder-DeWan S, et al. High-quality health systems in the Sustainable Development Goals era: time for a revolution. The Lancet Global Health. 2018;6(11):e1196-e252. 
24. Graham JL, Shahani L, Grimes RM, Hartman C, Giordano TP. The Influence of Trust in Physicians and Trust in the Healthcare System on Linkage, Retention, and Adherence to HIV Care. AIDS patient care and STDs. 2015;29(12):661-7.

25. Haywood C, Jr., Diener-West M, Strouse J, Carroll CP, Bediako S, Lanzkron S, et al. Perceived discrimination in health care is associated with a greater burden of pain in sickle cell disease. J Pain Symptom Manage. 2014;48(5):934-43.

26. Fiscella K, Franks P, Clancy CM. Skepticism toward medical care and health care utilization. Medical care. 1998;36(2):180-9.

27. Kruk ME, Gage AD, Joseph NT, Danaei G, García-Saisó S, Salomon JA. Mortality due to low-quality health systems in the universal health coverage era: a systematic analysis of amenable deaths in 137 countries. The Lancet. 2018;392(10160):2203-12.

28. Adler NE, Newman K. Socioeconomic Disparities In Health: Pathways And Policies. Health Affairs. 2002;21(2):60-76.

29. Salari P, Di Giorgio L, llinca S, Chuma J. The catastrophic and impoverishing effects of out-of-pocket healthcare payments in Kenya, 2018. 2019;4(6):e001809.

30. Sommer I, Griebler U, Mahlknecht P, Thaler K, Bouskill K, Gartlehner G, et al. Socioeconomic inequalities in non-communicable diseases and their risk factors: an overview of systematic reviews. BMC Public Health. 2015;15:914-.

31. Vedanthan R, Kamano JH, DeLong AK, Naanyu V, Binanay CA, Bloomfield GS, et al. Community Health Workers Improve Linkage to Hypertension Care in Western Kenya. J Am Coll Cardiol. 2019;74(15):1897-906.

32. Leung CL, Naert M, Andama B, Dong R, Edelman D, Horowitz C, et al. Human-centered design as a guide to intervention planning for non-communicable diseases: the BIGPIC study from Western Kenya. BMC health services research. 2020;20(1):415.

33. Leave no one behind: Strengthening health systems for UHC and the SDGs in Africa. Brazzaville, Republic of the Congo: WHO Regional Office for Africa 2017.

\section{Figures}




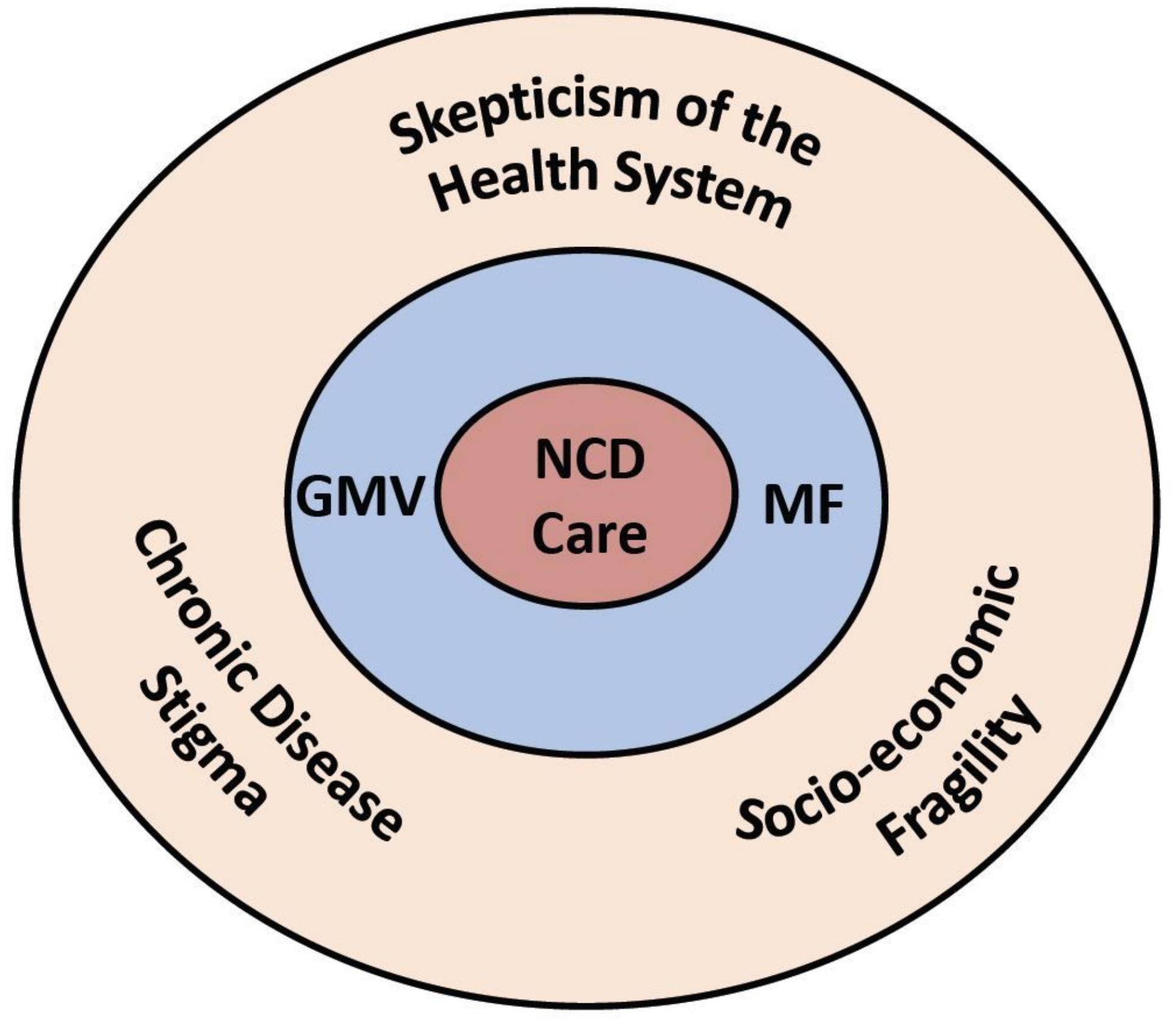

Figure 1

Conceptual representation of challenges to NCD care that can be mitigated by GMV and MF.

\section{Supplementary Files}

This is a list of supplementary files associated with this preprint. Click to download.

- BIGPICAim1CoverLetter20200714.docx

- StandardsforReportingQualitativeResearch20200630RV.docx 